\title{
Bacterial and Salivary Biomarkers Predict the Gingival Inflammatory Profile
}

Angie Lee, ${ }^{*}$ Carrie B. Ghaname, ${ }^{*}$ Thomas M. Braun, ${ }^{\dagger}$ James V. Sugai, $^{*}$ Ricardo P. Teles, ${ }^{\ddagger \S}$ Walter J. Loesche," Kenneth S. Kornman, "I William V. Giannobile, * and Janet S. Kinney*

Background: The aim of this human investigation is to explore the relationship of gingivitis with salivary biomarkers, periodontal pathogens, and interleukin (IL)-1 polymorphism after a transient inflammatory burden.

Methods: Thirty healthy human participants were randomized by IL-1 genotype status to control for potential influences of this particular single nucleotide polymorphism on the inflammatory profile. Oral hygiene practices ceased for 21 days to induce gingivitis (induction), after which home care was reinstated until 35 days (resolution). Clinical parameters included plaque (PI) and gingival (GI) indices and papillary bleeding score (PBS). Levels and proportions of 40 subgingival bacteria were determined using checkerboard DNA-DNA hybridization. Saliva was analyzed using a multiplex protein array for 30 biomarkers associated with host defense, inflammation, tissue destruction, and angiogenesis.

Results: Mean PI, GI, and PBS values were significantly increased during induction and decreased during resolution as measured at 35 days $(P<0.01)$, although no differences were observed between IL-1 groups. Participants were stratified as either "high" or "low" responders based on inflammatory response (high: GI >1.5; low: GI $\leq 1.5$ ). Baseline levels of salivary IL- 6 and IL- 8 demonstrated the highest ability to discriminate between high and low responders (area under the curve [AUC] of 0.81 and 0.72 , respectively). Salivary biomarkers, matrix metalloproteinases (MMPs), and bacterial biofilm were combined to generate receiver operating characteristic curves. High levels of IL- 6 and MMP- 1 at baseline demonstrated the strongest ability to predict high responders (AUC of 0.89; odds ratio of 17.0; 95\% confidence interval, 1.7 to 171.7$)$.

Conclusion: In this proof-of-concept investigation, we identified specific biomarker and microbial signatures that are associated with gingival inflammation (ClinicalTrials.gov number NCT00980525). J Periodontol 2012;83:79-89.

\section{KEY WORDS}

Bacteria; biomarkers; genetic polymorphism; gingivitis; inflammation mediators; saliva.

\footnotetext{
* Department of Periodontics and Oral Medicine, Michigan Center for Oral Health Research, University of Michigan, Ann Arbor, MI.

$\dagger$ Biostatistics Department, School of Public Health, University of Michigan.

† Department of Periodontology, The Forsyth Institute, Cambridge, MA.

$\S$ Division of Periodontology, Department of Oral Infection and Immunity, Harvard School of Dental Medicine, Boston, MA.

\| Department of Biologic and Materials Sciences, School of Dentistry, University of Michigan.

II Interleukin Genetics, Waltham, MA.
}

G ingivitis is a local inflammatory response to an oral infection involving an average of three to four teeth that affects $\approx 50 \%$ of the adult population. ${ }^{1}$ Although dental plaque biofilm is the primary etiology of most periodontal diseases, patients are not equally susceptible and do not respond similarly to professional care. ${ }^{2}$ Multiple genetic and environmental risk factors predispose an individual to periodontitis, 3,4 but the influence of these factors on gingivitis is uncertain.

Although periodontal diseases are initiated by bacterial pathogens, it is the activation and mediation of host inflammatory responses that are ultimately responsible for the destructive events occurring in the periodontium. ${ }^{5}$ Studies of the host immune response to pathogenic bacteria have contributed to the current understanding of the pathogenesis of periodontal diseases. ${ }^{6,7}$

The experimental gingivitis model is widely used to study the gingival events and microbiology that occur during a bacterially induced transition from a state of health to inflammation because it provides a well-controlled environment to study the pathogenesis and treatment of gingivitis. ${ }^{8}$ Limitations of the experimental gingivitis model include interexaminer differences when measuring plaque (PI) and gingival (GI) indices and the 10- to 21 -day requirement for gingivitis to be clinically measured. ${ }^{8}$

Studies examining levels of proinflammatory and regulatory biomarkers in

doi: 10.1902/jop.2011.110060 


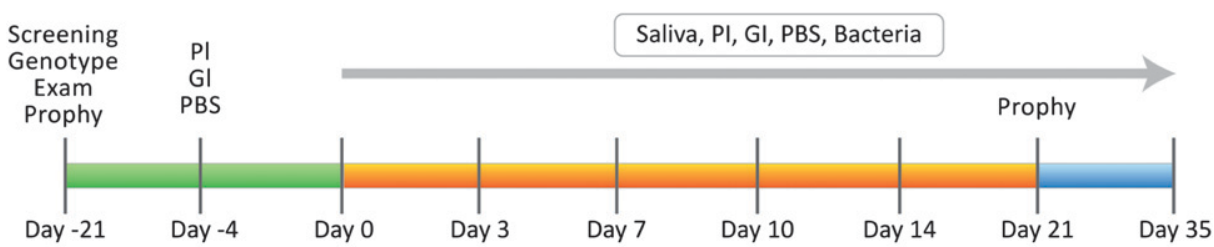

Pretrial Phase

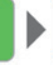

Experimental Gingivitis Phase

Figure I.

Timeline of the experimental gingivitis study. Genotype-periodontal susceptibility test; prophyprophylaxis; bacteria-subgingival plaque collection.

gingival crevicular fluid (GCF) and whole saliva of various disease states have provided insight to the diagnostic and prognostic value of these oral fluids. ${ }^{9-13}$ Putative biomarkers, such as cytokines and matrix metalloproteinases (MMPs), found in whole saliva, have been shown to correlate with actively progressing periodontal diseases. ${ }^{11}$ In fact, identification of select biomarkers combined with specific periodontal pathogens provides an even more accurate assessment and prediction of periodontal diseases than traditional clinical indices. ${ }^{14}$ Furthermore, when active sites in periodontally susceptible patients are recognized earlier, oral health care costs can be reduced. ${ }^{10}$

Although gingival fluid biomarker associations with experimental gingivitis have been reported, ${ }^{3}$ little is known about how the combination of a patient's levels of salivary biomarkers and presence of oral microorganisms might predict susceptibility to gingivitis. This study, therefore, seeks to determine whether salivary biomarkers and other biologic parameters at baseline might be predictive of "high" versus "low" responders to the bacterial challenge in the experimental gingivitis model, thereby allowing more objective determination of the gingival response and shorter runs of the model. Such information should be of high value in the search for technologies to control or prevent gingivitis.

\section{MATERIAL AND METHODS}

\section{Study Population}

Participants provided written informed consent to the protocol approved by the University of Michigan Health Sciences Institutional Review Board before administration of research-related procedures. The investigation was registered through National Institutes of Health ClinicalTrials.gov (identifier no. NCT00980525) and was conducted at the Michigan Center for Oral Health Research.

Because genetic differences influence monocyte expression and gingival fluid levels of interleukin (IL)$1 \beta, 7,15$ we compared experimental gingivitis responses in 15 participants who were IL-1 genotype positive and 15 participants who were IL- 1 genotype negative. Participants were volunteers recruited from February

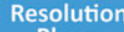

2009 to February 2010 and received monetary compensation for their participation.

White participants aged 18 to 40 years with $\geq 20$ permanent teeth were considered candidates. Inclusion criteria consisted of: 1) periodontal probing depth (PD) $\leq 4 \mathrm{~mm}$ at six sites per tooth on all teeth; 2) mean clinical attachment level (CAL) $\leq 2 \mathrm{~mm}$ on all teeth and mean PI, ${ }^{16} \mathrm{GI},{ }^{17}$ and papillary bleeding score ${ }^{18}$ (PBS) $\leq 1$ at the screening and confirmation appointment; and 3) compliance with all study requirements.

Participants were excluded if they: 1) were current smokers, quit smoking $<1$ year, or had a pack-year history $\geq 10$ (pack years was calculated by multiplying the number of years smoked by the average number of cigarette packs smoked per day); 2) had cotinine level $\geq 200 \mathrm{ng} / \mathrm{mL}$; 3 ) had antibiotic therapy within 3 months of baseline or need of prophylactic antibiotic; 4 ) had chronic consumption of medications known to affect the periodontal status; 5 ) were new oral contraceptives users within 3 months of baseline, or users who were planning on starting oral contraceptives or use of contraceptive injection ${ }^{\#}$ during the study; 6) were pregnant or lactating; 7) had current orthodontic or periodontal treatments; 8) had history of alcoholism or drug abuse; 9) had untreated carious lesions or defective restorations that could exacerbate during oral hygiene abstinence; or 10) had diseases of the immune system, or any medical condition that may influence the outcome.

\section{Clinical Procedures}

The timeline shown in Figure 1 summarizes the details of each appointment. Three weeks before baseline (i.e., screening), qualifying participants $(n=61)$ received a complete periodontal examination, prophylaxis, oral hygiene instructions, and topical fluoride application. Full-mouth PI, ${ }^{16} \mathrm{GI},{ }^{17} \mathrm{PD}$, and CAL were recorded using a periodontal probe. ${ }^{*} \mathrm{PBS}^{18}$ was measured using a triangular-shaped toothpick. ${ }^{\dagger \dagger}$ Participants provided a urine specimen to confirm their non-smoking status via cotinine levels $<200 \mathrm{ng} / \mathrm{mL}$. All participants refrained from using anti-inflammatory toothpaste between screening and confirmation visits.

Seventeen days after the initial visit (i.e., confirmation appointment; day -4), compliance was reassessed by measure of full-mouth PI, GI, and PBS to

\# Depo-Provera, Pharmacia \& Upjohn Company, Division of Pfizer, New York, NY.

** University of North Carolina Dental Probe, PCP-UNC 15, Hu-Friedy, Chicago, IL.

$\dagger \dagger$ Stim-U-Dent, Johnson \& Johnson, New Brunswick, NJ. 
ensure maximal gingival health. Participants with mean PI, GI, or PBS $>1$ were excluded. During the induction phase (i.e., day 0 to day 21 ), all measures of oral hygiene were discontinued, and participants were instructed to refrain from using mouth rinses or chewing gums with anti-inflammatory properties. At day 21 , a professional prophylaxis and topical fluoride were provided. During the resolution phase (i.e., day 21 to day 35), oral hygiene procedures were reinstituted. Participants were reevaluated at day 35 to ensure reestablishment of gingival health. Saliva, clinical measurements, and bacterial plaque were collected at days $0,3,7,10,14,21$, and 35. Clinical measurements included PI and GI on four randomized teeth and full-mouth PBS.

Three examiners (AL, JSK, and CBG) calibrated on PD and CAL before the beginning of the study. Examiners demonstrated at least $96 \%$ of PD measurements within $1 \mathrm{~mm}$ of each other with a $95 \%$ confidence interval of $(0.88,0.99)$ and at least $93 \%$ of CAL measurements within $1 \mathrm{~mm}$ of each other with a $95 \%$ confidence interval of $(0.84,0.97)$. Examiners completed training and calibration on PI, GI, and PBS. Examiners and participants were masked to IL-1 genotype results.

\section{IL-1 Genotype Analysis}

A genetic test was used to identify participants who had specific variations in the ILIA and IL1B genes. Buccal cells were obtained from participants by brushing their cheek with a cytology brush. ${ }^{\neq}$Brushes were air dried and mailed to a Clinical Laboratory Improvement Amendments-certified laboratory $§ \S$ for DNA extraction and genotyping.

The polymorphic loci assessed were IL $1 A+4845$ (rs17561) and IL1B + 3954 (rs1143634). Single nucleotide polymorphisms (SNPs) were genotyped by polymerase chain reactions targeting the sequence surrounding SNPs studied. Multiplexed single-base extension reactions were performed, genotypes were analyzed, |||| and final machine-determined genotypes were verified by a laboratory technologist masked to all participant data. Patients with composite genotypes that included $(I L 1 A+4845=$ guanine/thymine or thymine/thymine) and (IL1B + $3954=$ cytosine/ thymine or thymine/thymine) were classified as "positive" for increased risk for more severe chronic periodontitis.

\section{Whole Saliva Collection and Analysis}

Unstimulated whole saliva was collected from each participant at the beginning of the appointment and stored at $-80^{\circ} \mathrm{C}$ until analysis, as described previously by Kinney et al. ${ }^{14}$

Inflammatory biomarker expression was quantified using a custom human 30-plex protein array that was optimized for sensitivity, specificity, stability, and intraassay coefficient of variation by comparing to single cytokine enzyme-linked immunosorbent assays. "10 The 30-biomarker panel consisted of Creactive protein, Fcr RIIB/C, granulocyte colony stimulating factor (GCSF), interferon- $\gamma$ (IFN- $\gamma$ ), IL- $1 \alpha$, IL-1 $\beta$, IL-1ra, IL-1sRI, IL-2, IL-6, IL-8, IL-10, IL-12 (p40), IL-12 (p70), IL-17, interferon-induced protein10 (IP-10), monocyte chemoattractant protein-1 (MCP-1), macrophage inflammatory protein (MIP)$1 \alpha$, MIP-1 $\beta$, MMP-1, MMP-2, MMP-8, MMP-9, MMP10 , regulated on activation normal $T$-cell expressed and secreted protein, serum amyloid A, stromal cellderived factor- $1 \alpha$, tissue inhibitor of MMP (TIMP)-1, TIMP-2, and tumor necrosis factor- $\alpha$.

After receipt, the array kits were stored at $-20^{\circ} \mathrm{C}$. Before each assay, whole saliva samples were thawed at room temperature and microcentrifuged at 8000 to $10,000 \times \mathrm{g}$ for 5 minutes to obtain a cell-free supernatant.

Each slide contained known concentration of standards (in picograms per milliliters) for each cytokine, used for making serial dilutions to yield a six-point standard curve, with sample diluent serving as the negative control. Standards and experimental samples were incubated overnight at $4^{\circ} \mathrm{C}$, followed by washing unbound materials. Detection antibody was bound to antigens within each well. Cyanine 3 equivalent dye-conjugated streptavidin was pipetted into each well, which bound to the detection antibody associated with immune complexes. Slides were incubated, and fluorescence intensity was detected using a laser scanner."\# Resultant signals of samples were compared to the standard curve for each of the 30 cytokines to determine concentrations of each cytokine within the samples. Data were extracted and analyzed using microarray analysis software. ***

\section{Subgingival Bacterial Isolation and Analysis}

After collection of saliva and measurement of clinical parameters, subgingival plaque was collected from the mesio-buccal surface of four randomly selected teeth. A randomization chart was created to ensure that each site was sampled only once.

After removal of supragingival plaque, subgingival plaque samples were collected using sterile curets. ${ }^{\dagger \dagger}$ Samples were placed into labeled vials containing $150 \mu \mathrm{L}$ of Tris-EDTA buffer $(10 \mathrm{mM}$ Tris- $\mathrm{HCl}, 1 \mathrm{mM}$ EDTA, and $500 \mathrm{~mL}$ distilled water [pH 7.6]), after which $100 \mu \mathrm{L}$ of $0.5 \mathrm{M} \mathrm{NaOH}$ was

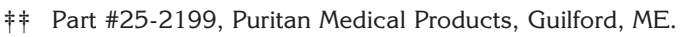

$\S \S$ Interleukin Genetics, Waltham, MA.

III CEQ8800 Instrument, Beckman Coulter, Brea, CA

II Quantibody Custom Array, RayBiotech, Norcross, GA

\#\# Axon Gene Pix, RayBiotech.

*** RayBio Q Analyzer software, RayBiotech.

$\dagger \dagger \dagger$ Gracey curets, Hu-Friedy.
} 


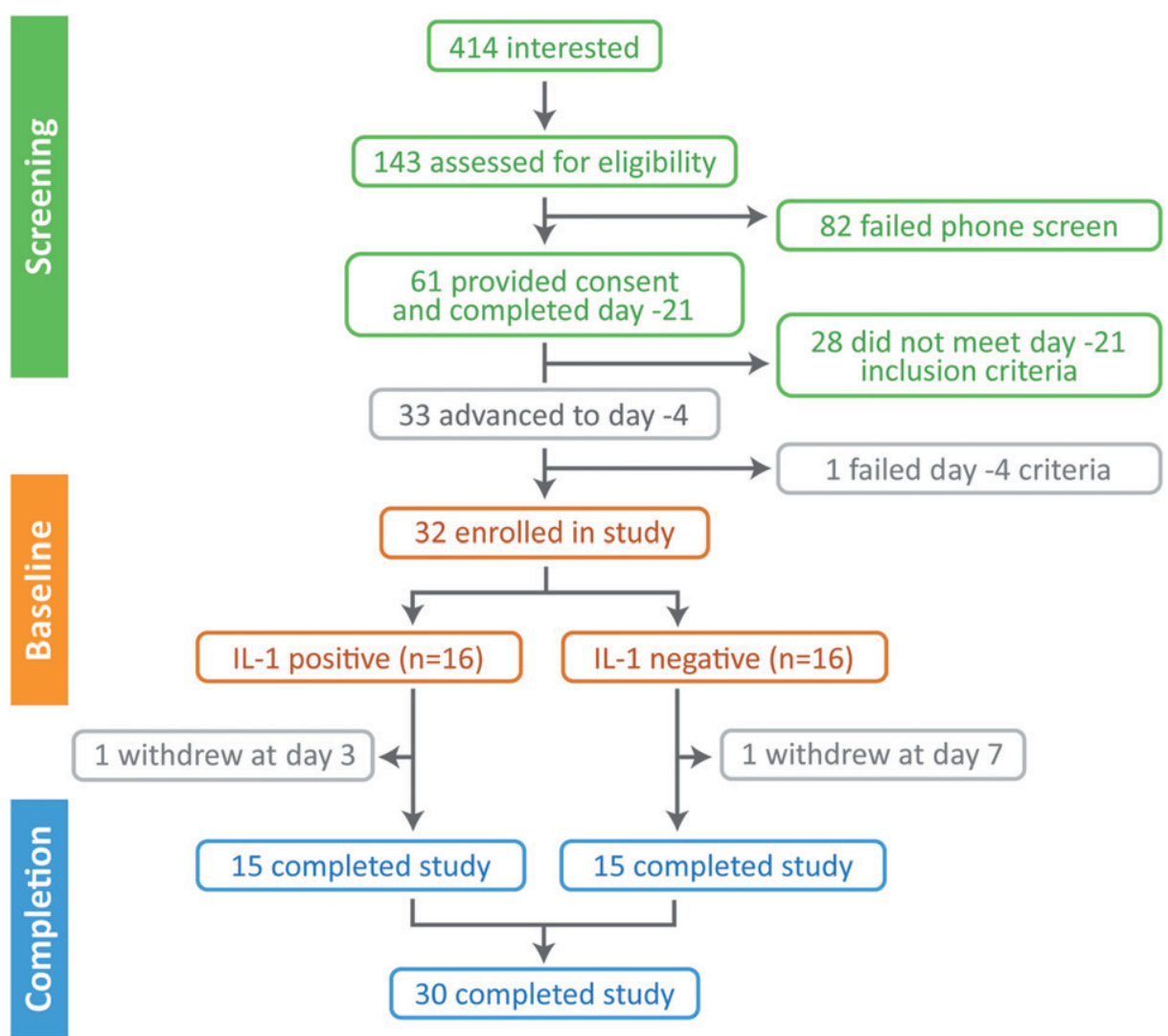

Figure 2.

Patient flowchart illustrating participant recruitment and disposition.

added to each vial. Samples were stored at $4^{\circ} \mathrm{C}$ until processed.

Detection of 40 bacterial species was evaluated by checkerboard DNA-DNA hybridization technique. ${ }^{19}$ Samples were lysed, and DNA was pipetted on a nylon membrane using a miniblotter apparatus. ${ }^{\ddagger} \neq$ After fixation of DNA to the membrane, the membrane was placed in another blotting apparatus,,$\S \S$ with lanes perpendicular to the orientation of the DNA of the samples. Digoxigenin-labeled whole chromosomal DNA probes to 40 subgingival species were loaded in individual lanes of the miniblotter. After hybridization, membranes were washed at low and high stringency. Hybrids were detected using anti-digoxigenin antibody and conjugated with alkaline phosphatase, followed by detection using a chemifluorescence substrate. IIIII The intensity of fluorescence signals generated by the probe-target hybridization was read with computer-linked fluorimager. "ItTl Each run contained two lanes of standards with a concentration of $10^{5}$ and $10^{6}$ cells of each species. Sensitivity of the assay and concentration of each DNA probe were adjusted to allow detection of $\geq 10^{4}$ cells of a given species. Signals were compared to standards on the same membrane to generate absolute counts. The inability to detect a signal was recorded as zero.

\section{Statistical Analyses}

Differences in demographic characteristics and mean clinical parameters between IL-1 genotype-positive and genotype-negative participants were assessed using $t$ tests for continuous measures and $\chi^{2}$ tests of association for categorical measures.

All microbiologic, salivary, and clinical outcomes were averaged within each participant before being analyzed. For certain analyses, summary measures for six subgingival complexes described by Socransky et al. ${ }^{20}$ were obtained by adding the mean values of each member of a given complex. Significance of changes over time in proportions of microbial complexes was determined using the Friedman test. Significance of differences in microbial counts between groups was tested using the Mann-Whitney $U$ test, whereas significance of changes over time in each group separately was sought using the Friedman test.

Participants were stratified into high and low responders such that high responders were characterized by a mean GI $>1.5$ at any time point between day 3 and day 21. Low responders were defined as participants with a mean GI $\leq 1.5$ at all time points between day 3 and day 21. Levels of day 0 salivary biomarkers and periodontal pathogens were ranked by their area under the curve $(A \cup C)$ of the receiver operating characteristic (ROC) curve for discriminating between high and low responders. Biomarkers that gave the highest AUCs were selected in the hopes of generating a composite ROC curve with higher AUC than each of its components.

Composite ROC curves were generated by combining the values of three biomarkers at baseline in a logistic regression model predicting for progression of GI $>1.5$. Each biomarker was classified individually as being high in a participant if that participant's value was above the value that produced equal values of

キキ MiniSlot, Immunetics, Boston, MA.

$\S \S \S$ Miniblotter 45, Immunetics.

IIII AttoPhos, GE Healthcare, Arlington Heights, IL.

ๆๆๆ Storm Fluorimager, GE Healthcare, Sunnyvale, CA. 
sensitivity and specificity. Odds ratios were used to determine odds of progression of GI $>1.5$ in participants high in all three biomarkers relative to participants not high in all three biomarkers. Standard errors (SEs) were computed using generalized estimating equations to account for the correlation of values measured longitudinally on the same participant. All $P$ values were adjusted for multiple comparisons between IL-1 genotype-positive and genotype-negative patients so as to reduce the rate of false-positive findings.

\section{RESULTS}

A flowchart of participants in the study is provided (Fig. 2). One hundred forty-three participants were screened for eligibility. Eighty-two participants failed the phone screen, and 28 did not meet study inclusion criteria. Two participants withdrew because of their inability to comply with abstinence of oral hygiene. Minor transient adverse events were reported and included soft and hard tissue sensitivity, altered taste sensation, and traumatic ulcer formation.

Fifteen participants who were IL-1 genotype positive (10 males and five females; mean age: $23.9 \pm$ 3.4 years) and 15 participants who were IL- 1 genotype negative (five males and 10 females; mean age: $23.9 \pm 5.3$ years) completed the study. Clinical and demographic information of the patients at baseline stratified according to IL- 1 genotype was analyzed, and no significant differences were observed between the two groups $(P>0.05)$ (see Supplementary Table 1 in online Journal of Periodontology).

\section{Clinical, Microbial, and Biomarker Changes With Induction of Gingivitis}

During the induction phase, a statistically significant increase in microbial accumulation and subsequent inflammation was observed in IL-1 genotype-positive and genotype-negative groups, as demonstrated by PI, GI, and PBS, although no significant differences were observed between the two groups (Fig. 3). Mean PI ( \pm SE) increased significantly from $0.38 \pm 0.04$ at baseline to $1.41 \pm 0.04$ at day 21 ( $P$ $<0.01)$ (Fig. 3A). Mean GI ( \pm SE) significantly increased from $0.32 \pm 0.05$ at baseline to $1.97 \pm$ 0.08 at day $21(P<0.01)$ (Fig. 3B). Mean \pm SE PBS increased from $0.31 \pm 0.04$ at baseline to $1.34 \pm$ 0.05 at day $21(P<0.01)$ (Fig. $3 C)$. All clinical parameters significantly decreased from day 21 to day $35(P<0.05)$.

Total bacterial counts increased significantly from baseline to day 21 and decreased significantly from day 21 to day $35(P<0.05)$ (Fig. 4A). As the experimental gingivitis phase progressed, the proportion of orange complex increased, with the most promi- nent peak at day 21. Mean counts of Prevotella melaninogenica at day 10 were significantly higher in IL-1 genotype-positive participants compared to the IL-1 genotype-negative group $(P=0.001)$ (Fig. 4B).

IL-1 $\beta$ at day 35 was the salivary biomarker that was significantly elevated in IL-1 genotype-positive participants compared to genotype-negative $(P=0.004)$ (Fig. 5). IL-1 genotype-positive participants had consistently higher levels of IL- $1 \alpha$ and IL- $1 \beta$ at all time points (Fig. 5). In IL-1 genotype-positive participants, salivary IL-1 $\beta$, IL-10, and TIMP-1 levels increased during the induction phase, with a decrease of IL-2 and IL- 6 levels at resolution. A decrease in the levels of GCSF, IFN- $\gamma$, IL-1 $\alpha$, IL-1 $\beta$, IL-1ra, IL-2, IL-6, IL-8, IP-10, MCP-1, MMP-2, MMP-8, MMP-9, TIMP-1, and TIMP-2 was observed in IL-1 genotype-negative participants at day 10 , after which the biomarkers increased again, although not statistically significantly (see Supplementary Table 2 in online Journal of Periodontology). Most biomarkers returned to baseline levels at day 35.

\section{Prediction of Gingivitis Response}

Twelve participants belonged to the high responders group (six genotype-positive and six genotype-negative participants), whereas 18 participants belonged to the low responders group (nine genotype-positive and nine genotype-negative participants).

Of the proinflammatory salivary biomarkers, baseline IL- 6 and IL- 8 had the highest AUCs of 0.81 and 0.72 , respectively. Salivary baseline MMP-1 and MMP-8 demonstrated the highest AUCs of 0.76 and 0.78 , respectively. Subgingival bacterial baseline levels of Fusobacterium nucleatum polymorphum had the highest bacterial AUC of 0.75 , predicting high versus low responders, followed by Fusobacterium periodonticum with an AUC of 0.73 , Gemella morbillorum with an AUC of 0.74, and Capnocytophaga ochracea with an AUC of 0.72 .

Figure 6 depicts the AUCs and odds ratios for various combinations of salivary proinflammatory cytokines, MMPs, and bacterial pathogens. The composite of IL- 6 and MMP-1 generated an AUC of 0.89, and participants with high levels of both biomarkers at baseline had 17.0 times the odds (95\% confidence interval, 1.7 to 171.7 ) of being a high responder than participants without high levels of both biomarkers (Fig. 6).

\section{DISCUSSION}

The IL-1 family of cytokines is produced primarily by macrophages and is largely responsible for initiating the cascade of inflammatory responses. ${ }^{21}$ Genetic variations in the IL-1 gene cluster are associated with a hyperinflammatory state ${ }^{15}$ that increases susceptibility to periodontal diseases. ${ }^{4,22-26}$ 



Figure 3.

Longitudinal plots of mean \pm SE clinical periodontal measures of all participants from day -2I to day 35. A) PI. B) GI. C) PBS. Statistically significant increases were found during induction and decreases during resolution for all parameters $(\mathrm{P}<0.01)$.

The aims of this research are to understand how biomarkers and periodontal pathogens are modulated during a bacterially induced gingival inflammation and to use these findings to identify individuals with a more exuberant gingivitis response. The presence of 30 biomarkers associated with host defense, inflammation, tissue destruction, and angiogenesis was analyzed in saliva. Modulation of these genes and pathways has been demonstrated in a previous experimental gingivitis study. ${ }^{27}$ Our study provides additional support to a previous investigation exploring modulation of genetic pathways in experimental gin- givitis combining proteomic and microbial genetic biomarkers to differentiate between various forms of periodontal diseases ${ }^{14}$ and extends previous findings in two areas: 1) we demonstrate the effective use of saliva to monitor critical gingivitis biomarkers; and 2) we show that a combination of objective baseline parameters is highly predictive of high responders to the acute bacterial challenge.

Our study controlled for multiple variables that influence gingival inflammation. However, a lack of sex balance between the two genotype groups was observed. Our study was powered to assess overall biomarker patterns during experimental gingivitis. However, our study was not powered to detect significant differences between IL-1 genotypes because of the relatively small effects of this gene compared to the dominant effects of bacterial accumulations associated with discontinuance of oral hygiene.

The polymorphic loci assessed in our study were IL-1A +4845 and IL-1B +3954 . Hence, a patient considered IL-1 genotype positive had a composite genotype that included both SNPs. However, IL-1B gene transcription is a much more complex process that involves different SNPs of the enhancer-promoter region of the IL-1B gene. Functional polymorphism of the IL-1B gene encompasses four functionally active SNPs in the promoter region: $-31,-511,-1464$, and $-3737 .{ }^{28}$ Because these SNPs constantly interact with each other, examination of the combination of alleles at different loci that are transmitted together (i.e., haplotypes) provides a much more compelling association with clinical phenotypes. Recent studies found that the combination of various functional IL-1B haplotypes displayed different levels of biologic activity and were significantly associated with IL-1 $\beta$ secretion. ${ }^{28,29}$ Future studies examining IL- 1 genotype should be directed at the correlation of these pairs of haplotypes with various disease phenotypes, such as elevated levels of salivary biomarkers and periodontal pathogens. Studies of haplotype pairs could possibly explain the inconsistency of the clinical application of testing for IL-1 genotype and differences among various ethnic populations. ${ }^{28,30-32}$

The clinical and microbial responses to cessation of oral hygiene were consistent with previous observations. ${ }^{8,33} \mathrm{~A}$ decrease in the levels of multiple biomarkers was observed in IL-1 genotype-negative participants at day 10 , after which the biomarkers rebounded. Downregulation of biomarkers, such as IL- $1 \beta$ and IL- 8 , in the early stages of gingivitis was reported in previous in vitro ${ }^{34}$ and experimental gingivitis studies. ${ }^{35,36}$ Some authors proposed that initially downregulated proinflammatory biomarkers can suddenly rise and predispose participants to an exaggerated inflammatory response. ${ }^{36-38}$ 
A
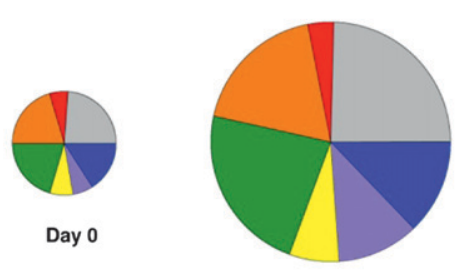

Day 3



Day 10

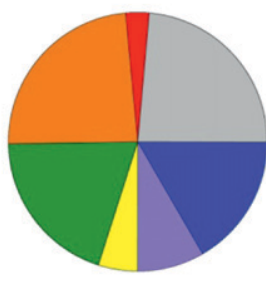

Day 14

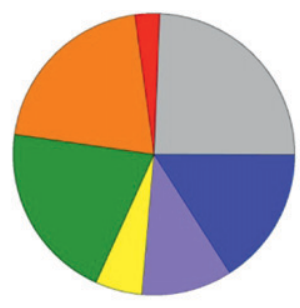

Day 7



Day 21
B

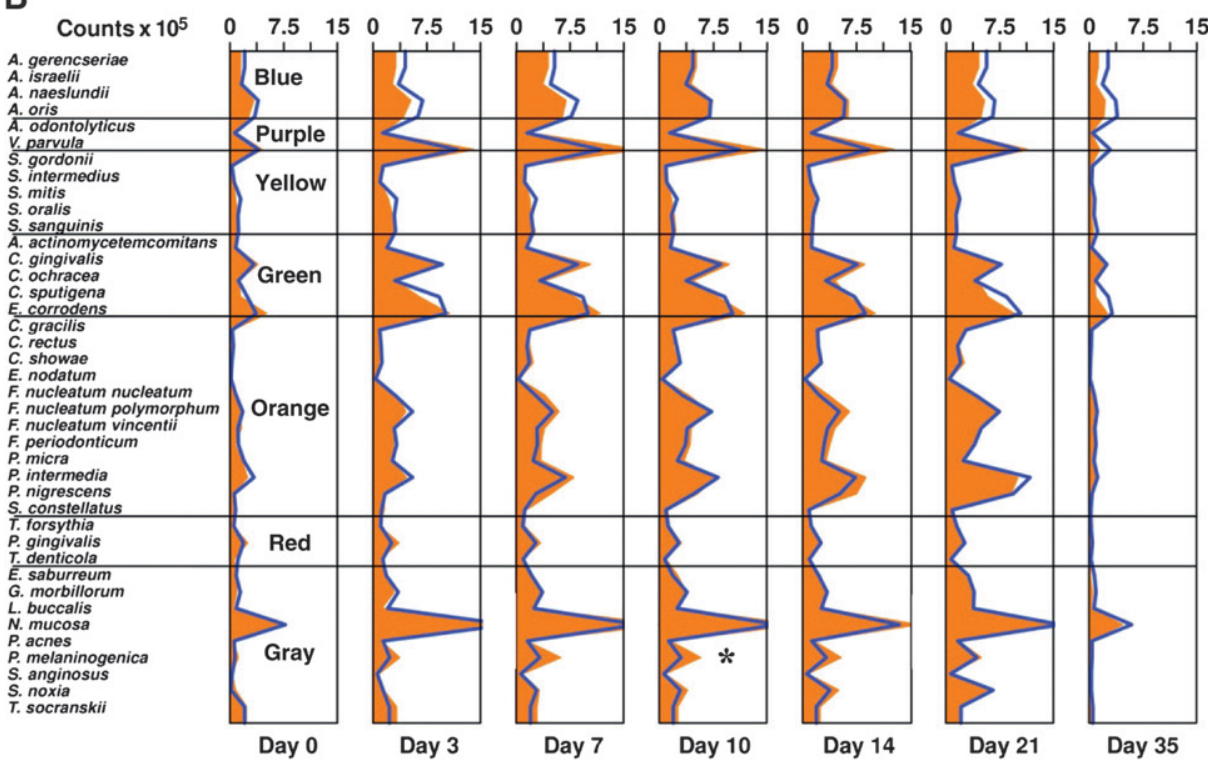

Figure 4.

A) Pie charts demonstrating proportions of each bacterial complex as defined by Socransky et al. ${ }^{20}$ in relation to total DNA from day 0 to day 35 . The size of the pies was adjusted to reflect the total DNA probe count. B) Mean counts $\left(\times 10^{5}\right)$ of 40 bacterial species stratified by IL-I genotype. Blue line delineates bacterial counts in IL-I genotype-positive participants. Orange fill represents bacterial counts in IL-I genotype-negative participants. ${ }^{*} \mathrm{P}<0.007$.

However, in our study, it appears that this downregulation occurred preferentially on IL-1-negative participants, suggesting a protective mechanism against inflammation. For example, although IL-8 plays a role in bacterial destruction, a hyperactivity of this cytokine increases periodontal tissue destruction by increasing additional neutrophilic infiltration and MMP- 8 production. ${ }^{39-41}$ As a result, an initial downregulation of proinflammatory cytokines may help limit host tissue degradation. This protective concept is further supported by a study in which periodontally healthy participants displayed significantly higher GCF IL-8 gate with most bacteria in the are high responders reinforces their role in facilitating coaggregation between early colonizers in gingivitis and periodontal pathogens in periodontitis. ${ }^{46}$ In fact, Fusobacterium species produce an environment favorable for growth of anaerobic pathogens by coaggregating with facultative organisms that consume the surrounding oxygen. ${ }^{46}$ Moreover, C. ochracea as a secondary colonizer produces succinate that can be used by Porphyromonas gingivalis to enhance its growth. ${ }^{47}$ Therefore, at baseline before the inflammatory burden, high responders possess 

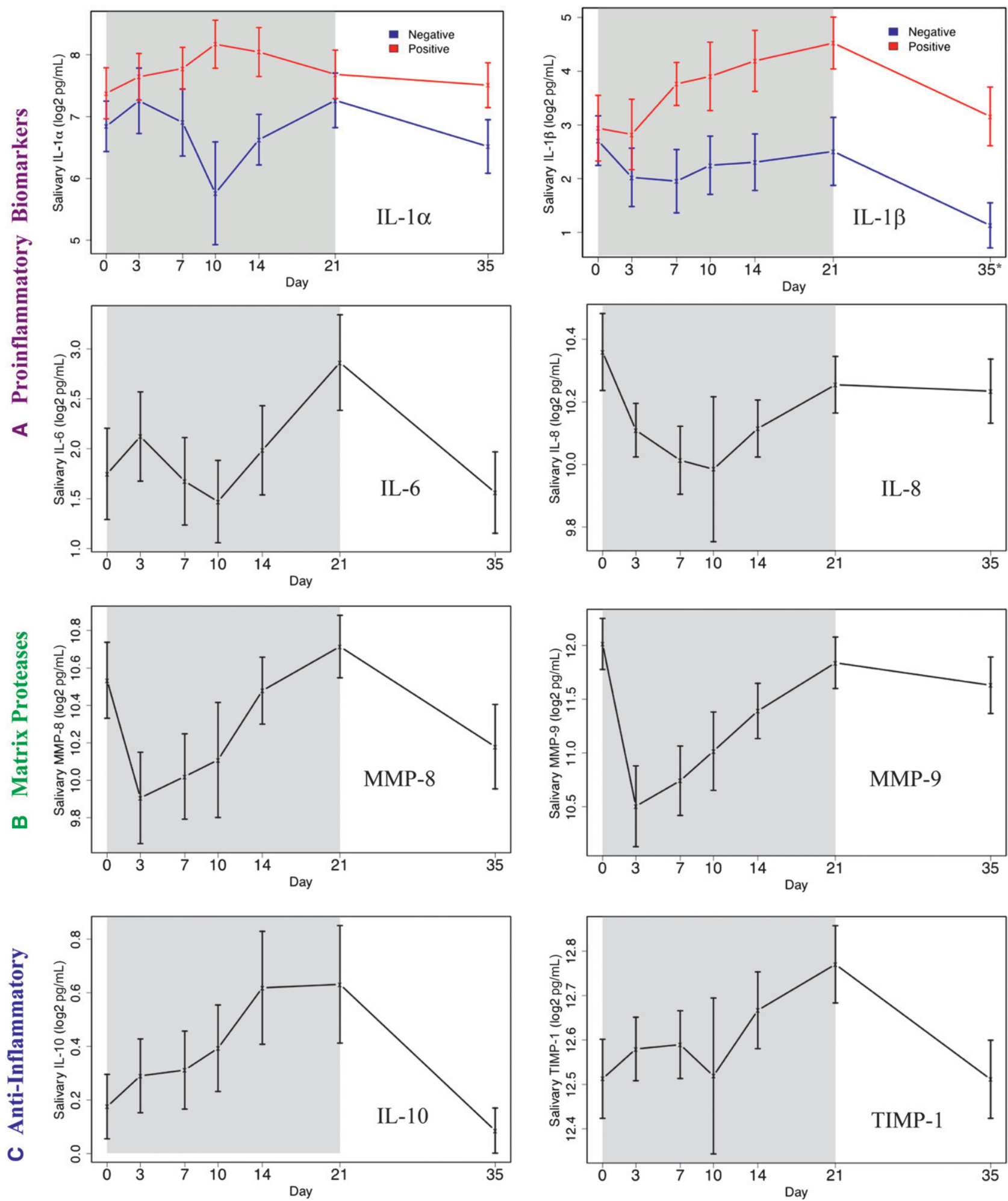

Figure 5.

Longitudinal plots of mean \pm SE salivary biomarkers from day 0 to day 35. A) Proinflammatory biomarkers stratified by IL-I genotype: IL-I $\alpha$ and IL-I $\beta$. Proinflammatory biomarkers of all participants: IL-6 and IL-8. B) MMPs of all participants: MMP-8 and MMP-9. C) Anti-inflammatory of all participants: IL-I 0 and TIMP-I. *P $<0.007$. 
A
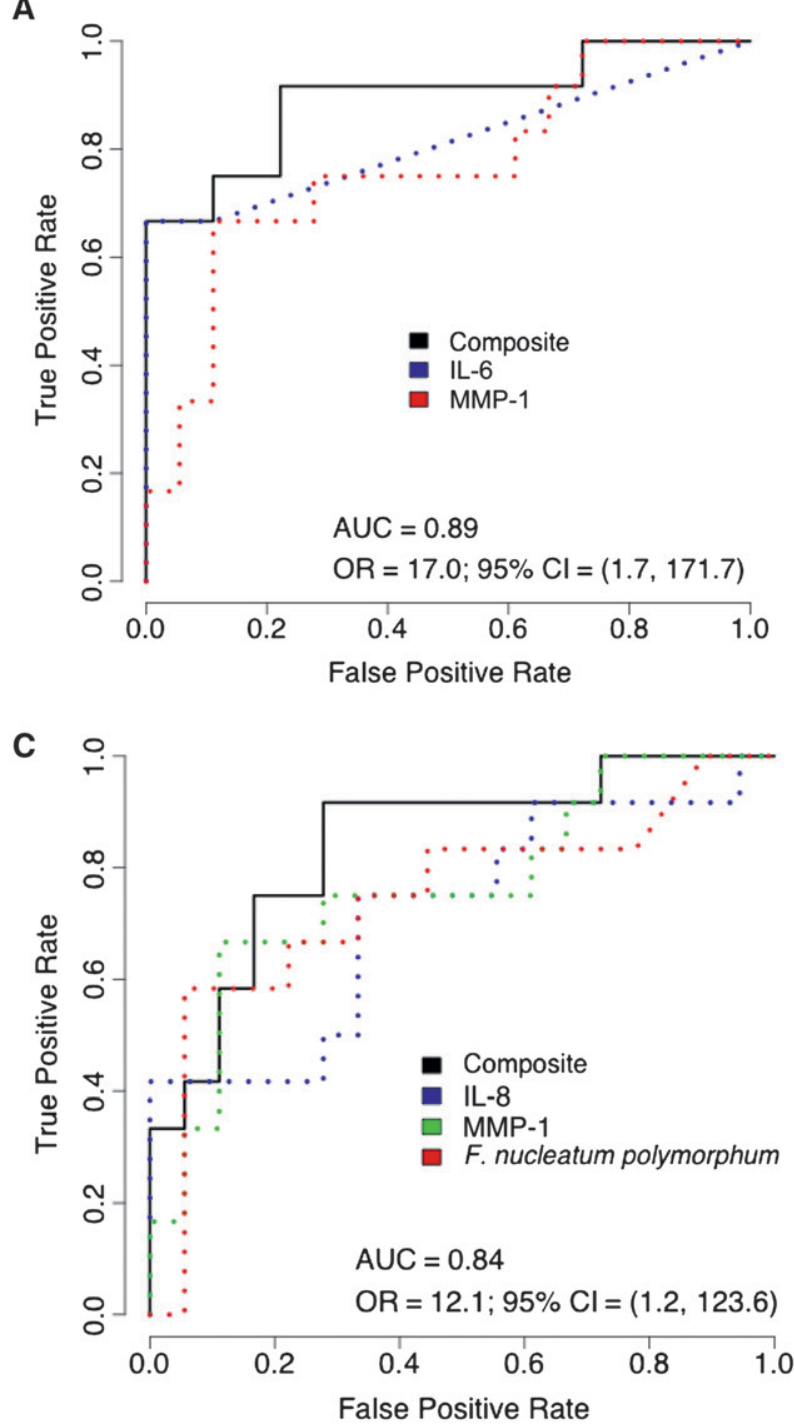

$\mathrm{E}$

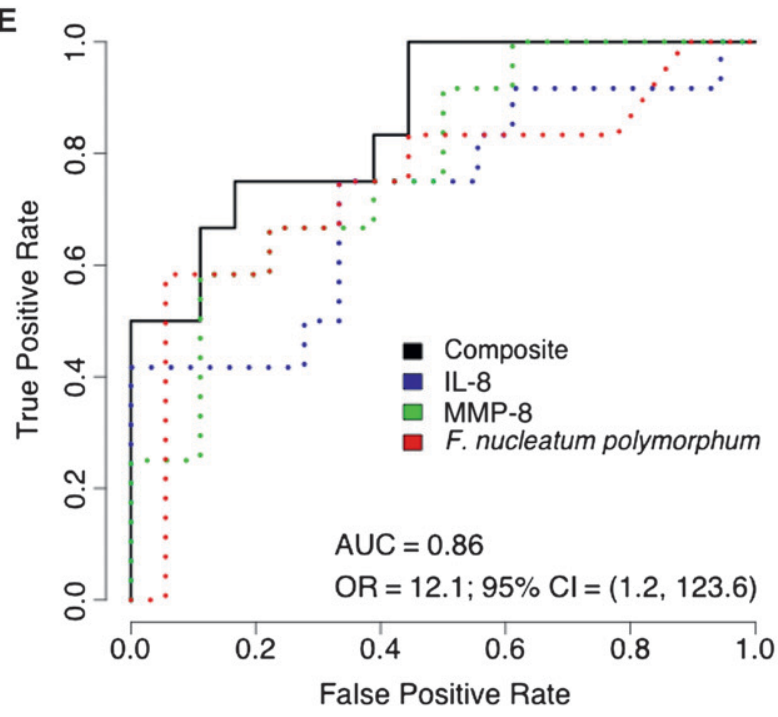

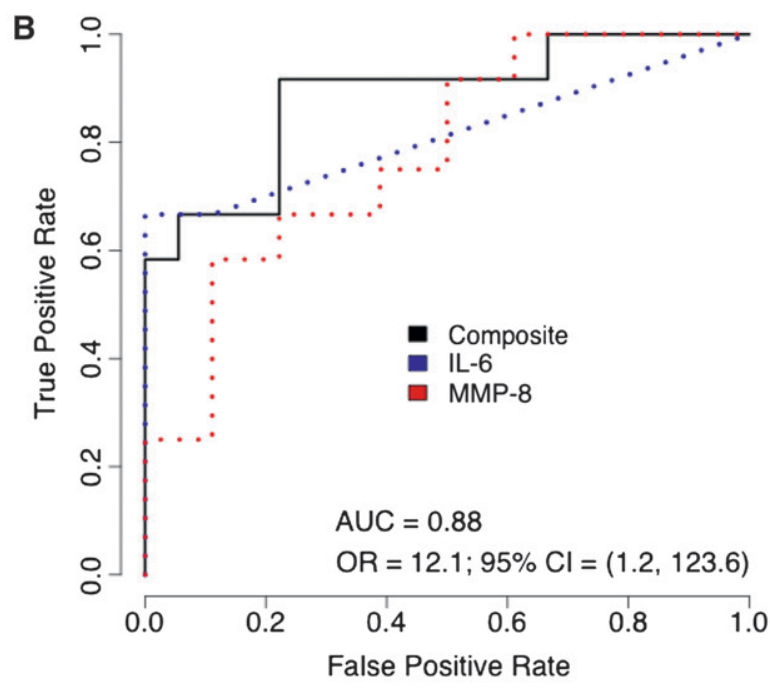
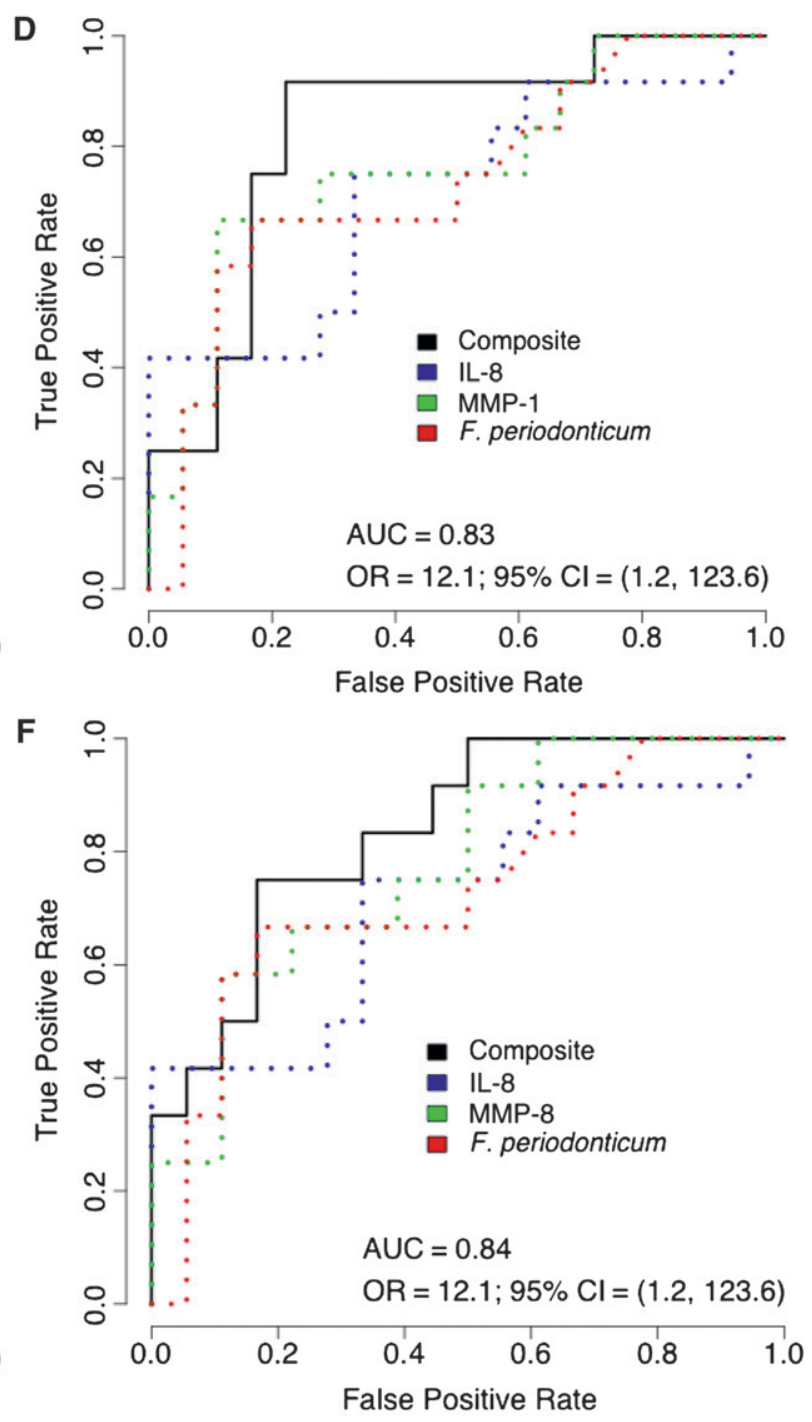

Figure 6.

ROC curves for IL-6 + MMP-I (A), IL-6 + MMP-8 (B), IL-8 + MMP-I + F. nucleatum polymorphum (C), IL-8 + MMP-I + F. periodonticum (D), IL-8 + MMP-8 + F. nucleatum polymorphum (E), and IL-8 + MMP-8 + F. periodonticum (F). Cl-Confidence interval; OR-odds ratio. 
a microbiologic profile that might facilitate colonization of periodontal pathogens.

The experimental gingivitis model consists of inducing an acute stage of inflammation to otherwise healthy patients, creating clinical and immunologic responses that are different from the naturally occurring chronic development of disease. ${ }^{7,36,48}$ In experimental gingivitis, participants receive a prophylaxis and subsequently refrain from all forms of oral hygiene for 21 days. In contrast, naturally occurring gingivitis patients still maintain some form of oral hygiene and develop gingivitis over a longer period of time. In a study by Deinzer et al., ${ }^{36}$ plaque accumulation and GCF IL-1 $\beta$ levels were significantly higher in experimental gingivitis compared to persistent gingivitis, whereas severity of gingival bleeding and GCF IL-8 levels were significantly lower. Moreover, experimental gingivitis lesions are dominated by a lymphocytic-dominated infiltrate rather than neutrophilic. ${ }^{49,50}$ Despite these differences, the acute inflammatory stress induced in experimental gingivitis has some similarities to active phases of destruction during chronic periodontitis. As a consequence, data in this study could reflect the microbiologic profile and biologic host response during the acute phases of inflammation. We also postulate that a subset of participants who displayed a biologic profile consistent with progressive periodontitis may have a cellular infiltrate or activation state more similar to periodontitis than gingivitis. For example, participants with high levels of IL- $1 \beta$ and MMP- 8 had 3.47 times the odds of being a high responder compared to participants with low levels of these biomarkers (data not shown). Whether such participants are more prone to progressive periodontitis when challenged remains to be determined.

\section{CONCLUSIONS}

Our study demonstrates that patients with high baseline levels of salivary IL- 6 and MMP- 1 are at strong risk of developing a heightened gingival inflammatory response compared to individuals displaying low levels of these biomarkers. Detection of these specific host and bacterial biomarkers using a practical chairside salivary test could allow early identification of susceptible patients so that a preventive intervention can be initiated before irreversible tissue breakdown. Future studies on large and diverse patient populations will be needed to validate these findings for application to the clinical arena.

\section{ACKNOWLEDGMENTS}

This work was supported by Colgate-Palmolive Company (Piscataway, NJ) Grant N011792-0, the Michigan Institute for Clinical Health Research (U026020), and National Institutes of Health/National Center for Research Resources Grant UL1RR024986
(J.S. Kinney). This trial was registered in the ClinicalTrials.gov database (identifier no. NCT00980525). The authors thank Lea Franco, Christina Huffman, Alice Liu, Amy Litch, Sooyeon Kwon, Anna Galloro, Chris Jung, and Andrei Taut at the Michigan Center for Oral Health Research, Ann Arbor, Michigan. Dr. Giannobile holds intellectual property related to the diagnostics technology described in this article. Dr. Kornman is chief scientific officer at Interleukin Genetics. Drs. Lee, Ghaname, Braun, Sugai, Teles, and Loesche report no conflicts of interest related to this study.

\section{REFERENCES}

1. Oliver RC, Brown LJ, Löe H. Periodontal diseases in the United States population. J Periodontol 1998;69: 269-278.

2. Kornman KS. Patients are not equally susceptible to periodontitis: Does this change dental practice and the dental curriculum? J Dent Educ 2001;65:777-784.

3. Salvi GE, Franco LM, Braun TM, et al. Pro-inflammatory biomarkers during experimental gingivitis in patients with type 1 diabetes mellitus: A proof-of-concept study. J Clin Periodontol 2010;37:9-16.

4. Kornman KS, Crane A, Wang HY, et al. The interleukin-1 genotype as a severity factor in adult periodontal disease. J Clin Periodontol 1997;24:72-77.

5. Kornman KS, Page RC, Tonetti MS. The host response to the microbial challenge in periodontitis: Assembling the players. Periodontol 2000 1997;14:33-53.

6. Offenbacher S, Barros S, Mendoza L, et al. Changes in gingival crevicular fluid inflammatory mediator levels during the induction and resolution of experimental gingivitis in humans. J Clin Periodontol 2010;37:324-333.

7. Trombelli L, Scapoli C, Carrieri A, Giovannini G, Calura G, Farina R. Interleukin-1 beta levels in gingival crevicular fluid and serum under naturally occurring and experimentally induced gingivitis. J Clin Periodontol 2010;37:697-704.

8. Löe H, Theilade E, Jensen SB. Experimental gingivitis in man. J Periodontol 1965;36:177-187.

9. Mandel ID. The diagnostic uses of saliva. J Oral Pathol Med 1990;19:119-125.

10. Giannobile WV, Beikler T, Kinney JS, Ramseier CA, Morelli T, Wong DT. Saliva as a diagnostic tool for periodontal disease: Current state and future directions. Periodontol 2000 2009;50:52-64.

11. Kinney JS, Ramseier CA, Giannobile WV. Oral fluidbased biomarkers of alveolar bone loss in periodontitis. Ann N Y Acad Sci 2007;1098:230-251.

12. Sorsa T, Tervahartiala T, Leppilahti J, et al. Collagenase-2 (MMP-8) as a point-of-care biomarker in periodontitis and cardiovascular diseases. Therapeutic response to non-antimicrobial properties of tetracyclines. Pharmacol Res 2011;63:108-113.

13. Leppilahti JM, Ahonen MM, Hernández M, et al. Oral rinse MMP- 8 point-of-care immuno test identifies patients with strong periodontal inflammatory burden. Oral Dis 2011;17:115-122.

14. Kinney JS, Morelli T, Braun T, et al. Saliva/pathogen biomarker signatures and periodontal disease progression. J Dent Res 2011;90:752-758.

15. Pociot F, Mølvig J, Wogensen L, Worsaae H, Nerup J. A TaqI polymorphism in the human interleukin-1 beta 
(IL-1 beta) gene correlates with IL-1 beta secretion in vitro. Eur J Clin Invest 1992;22:396-402.

16. Silness J, Löe H. Periodontal disease in pregnancy. II. Correlation between oral hygiene and periodontal condition. Acta Odontol Scand 1964;22:121-135.

17. Löe H, Silness J. Periodontal disease in pregnancy. I. Prevalence and severity. Acta Odontol Scand 1963; 21:533-551.

18. Loesche WJ. Clinical and microbiological aspects of chemotherapeutic agents used according to the specific plaque hypothesis. J Dent Res 1979;58:2404-2412.

19. Socransky SS, Haffajee AD, Smith C, et al. Use of checkerboard DNA-DNA hybridization to study complex microbial ecosystems. Oral Microbiol Immunol 2004; 19:352-362.

20. Socransky SS, Haffajee AD, Cugini MA, Smith C, Kent RL Jr. Microbial complexes in subgingival plaque. $J$ Clin Periodontol 1998;25:134-144.

21. Graves DT, Cochran D. The contribution of interleukin- 1 and tumor necrosis factor to periodontal tissue destruction. J Periodontol 2003;74:391-401.

22. Yoshie H, Kobayashi T, Tai H, Galicia JC. The role of genetic polymorphisms in periodontitis. Periodontol 2000 2007;43:102-132.

23. Kinane DF, Shiba H, Hart TC. The genetic basis of periodontitis. Periodontol 2000 2005;39:91-117.

24. Berger P, McConnell JP, Nunn M, et al. C-reactive protein levels are influenced by common IL-1 gene variations. Cytokine 2002;17:171-174.

25. McGuire MK, Nunn ME. Prognosis versus actual outcome. IV. The effectiveness of clinical parameters and IL-1 genotype in accurately predicting prognoses and tooth survival. J Periodontol 1999;70:49-56.

26. Lang NP, Tonetti MS, Suter J, Sorrell J, Duff GW, Kornman KS. Effect of interleukin-1 gene polymorphisms on gingival inflammation assessed by bleeding on probing in a periodontal maintenance population. $J$ Periodontal Res 2000;35:102-107.

27. Offenbacher S, Barros SP, Paquette DW, et al. Gingival transcriptome patterns during induction and resolution of experimental gingivitis in humans. $J$ Periodontol 2009;80:1963-1982.

28. Chen H, Wilkins LM, Aziz N, et al. Single nucleotide polymorphisms in the human interleukin-1B gene affect transcription according to haplotype context. Hum Mol Genet 2006;15:519-529.

29. Rogus J, Beck JD, Offenbacher S, et al. IL1B gene promoter haplotype pairs predict clinical levels of interleukin-1beta and C-reactive protein. Hum Genet 2008;123:387-398.

30. Huynh-Ba G, Lang NP, Tonetti MS, Salvi GE. The association of the composite IL-1 genotype with periodontitis progression and/or treatment outcomes: A systematic review. J Clin Periodontol 2007;34:305317.

31. Greenstein G, Hart TC. Clinical utility of a genetic susceptibility test for severe chronic periodontitis: A critical evaluation. J Am Dent Assoc 2002;133:452459; quiz 492-453.

32. Shapira L, Wilensky A, Kinane DF. Effect of genetic variability on the inflammatory response to periodontal infection. J Clin Periodontol 2005;32(Suppl. 6):72-86.

33. Loesche WJ, Syed SA. Bacteriology of human experimental gingivitis: Effect of plaque and gingivitis score. Infect Immun 1978;21:830-839.

34. Ohguchi Y, Ishihara Y, Ohguchi $M$, et al. Capsular polysaccharide from Actinobacillus actinomycetemco- mitans inhibits IL-6 and IL-8 production in human gingival fibroblast. J Periodontal Res 2003;38:191-197.

35. Zhang J, Kashket S, Lingström P. Evidence for the early onset of gingival inflammation following shortterm plaque accumulation. J Clin Periodontol 2002; 29:1082-1085.

36. Deinzer R, Weik U, Kolb-Bachofen V, Herforth A. Comparison of experimental gingivitis with persistent gingivitis: Differences in clinical parameters and cytokine concentrations. J Periodontal Res 2007;42:318-324.

37. Strömberg E, Edebo A, Lundin BS, et al. Downregulation of epithelial IL-8 responses in Helicobacter pylori-infected duodenal ulcer patients depends on host factors, rather than bacterial factors. Clin Exp Immunol 2005; 140:117-125.

38. Marks DJ, Harbord MW, MacAllister R, et al. Defective acute inflammation in Crohn's disease: A clinical investigation. Lancet 2006;367:668-678.

39. Jin L, Söder B, Corbet EF. Interleukin-8 and granulocyte elastase in gingival crevicular fluid in relation to periodontopathogens in untreated adult periodontitis. $J$ Periodontol 2000;71:929-939.

40. Tonetti MS, Freiburghaus K, Lang NP, Bickel M. Detection of interleukin- 8 and matrix metalloproteinases transcripts in healthy and diseased gingival biopsies by RNA/PCR. J Periodontal Res 1993;28:511-513.

41. Bickel $M$. The role of interleukin- 8 in inflammation and mechanisms of regulation. J Periodontol 1993; 64 (Suppl. 5):456-460.

42. Loesche WJ, Grossman NS. Periodontal disease as a specific, albeit chronic, infection: Diagnosis and treatment. Clin Microbiol Reu 2001;14:727-752.

43. Moore WE, Moore LV. The bacteria of periodontal diseases. Periodontol 2000 1994;5:66-77.

44. Listgarten MA, Lai CH, Young V. Microbial composition and pattern of antibiotic resistance in subgingival microbial samples from patients with refractory periodontitis. J Periodontol 1993;64: 155-161.

45. Moncla BJ, Braham P, Dix K, Watanabe S, Schwartz D. Use of synthetic oligonucleotide DNA probes for the identification of Bacteroides gingivalis. J Clin Microbiol 1990;28:324-327.

46. Kolenbrander PE. Coaggregation of human oral bacteria: Potential role in the accretion of dental plaque. J Appl Bacteriol 1993;74(Suppl.):79S-86S.

47. Grenier D, Mayrand D. Studies of mixed anaerobic infections involving Bacteroides gingivalis (in French). Can J Microbiol 1983;29:612-618.

48. Wahaidi VY, Kowolik MJ, Eckert GJ, Galli DM. Endotoxemia and the host systemic response during experimental gingivitis. J Clin Periodontol 2011;38:412-417.

49. Page RC, Schroeder HE. Pathogenesis of inflammatory periodontal disease. A summary of current work. Lab Invest 1976;34:235-249.

50. Seymour GJ, Powell RN, Aitken JF. Experimental gingivitis in humans. A clinical and histologic investigation. J Periodontol 1983;54:522-528.

Correspondence: Janet S. Kinney, Michigan Center for Oral Health Research, University of Michigan Clinical Center, 24 Frank Lloyd Wright Dr., Lobby M, Box 422, Ann Arbor, MI 48106. Fax: 734/763-5503; e-mail: kinneyj@umich.edu.

Submitted January 30, 2011; accepted for publication April 13, 2011. 